\title{
Management of latent Mycobacterium tuberculosis infection: WHO guidelines for low tuberculosis burden countries
}

\author{
Haileyesus Getahun ${ }^{1}$, Alberto Matteelli ${ }^{1}$, Ibrahim Abubakar ${ }^{2,3}$, \\ Mohamed Abdel Aziz ${ }^{4}$, Annabel Baddeley ${ }^{1}$, Draurio Barreira ${ }^{5}$, Saskia Den Boon ${ }^{6}$, \\ Susana Marta Borroto Gutierrez ${ }^{7}$, Judith Bruchfeld ${ }^{8}$, Erlina Burhan? \\ Solange Cavalcante ${ }^{10}$, Rolando Cedillos ${ }^{11}$, Richard Chaisson ${ }^{12}$, \\ Cynthia Bin-Eng Chee ${ }^{13}$, Lucy Chesire ${ }^{14}$, Elizabeth Corbett ${ }^{15}$, Masoud Dara ${ }^{16}$, \\ Justin Denholm ${ }^{17}$, Gerard de Vries ${ }^{18}$, Dennis Falzon ${ }^{1}$, Nathan Ford ${ }^{19}$, \\ Margaret Gale-Rowe ${ }^{20}$, Chris Gilpin ${ }^{1}$, Enrico Girardi ${ }^{21}$, Un-Yeong Go ${ }^{22}$ \\ Darshini Govindasamy ${ }^{23}$, Alison D. Grant ${ }^{24}$, Malgorzata Grzemska ${ }^{1}$, Ross Harris ${ }^{3}$, \\ C. Robert Horsburgh $\mathrm{Jr}^{25}$, Asker Ismayilov ${ }^{26}$, Ernesto Jaramillo ${ }^{1}$, Sandra Kik ${ }^{27}$, \\ Katharina Kranzer ${ }^{24}$, Christian Lienhardt ${ }^{1}$, Philip LoBue ${ }^{28}$, Knut Lönnroth ${ }^{1,29}$, \\ Guy Marks ${ }^{30}$, Dick Menzies ${ }^{27}$, Giovanni Battista Migliori ${ }^{31}$, Davide Mosca ${ }^{32}$, \\ Ya Diul Mukadi ${ }^{33}$, Alwyn Mwinga ${ }^{34}$, Lisa Nelson ${ }^{19}$, Nobuyuki Nishikiori ${ }^{35}$, \\ Anouk Oordt-Speets ${ }^{36}$, Molebogeng Xheedha Rangaka ${ }^{2,37}$, Andreas Reis ${ }^{38}$, \\ Lisa Rotz ${ }^{28}$, Andreas Sandgren ${ }^{39}$, Monica Sañé Schepisi ${ }^{21}$, Holger J. Schünemann ${ }^{40}$, \\ Surender Kumar Sharma ${ }^{38}$, Giovanni Sotgiu ${ }^{39}$, Helen R. Stagg ${ }^{2}$, \\ Timothy R. Sterling ${ }^{43}$, Tamara Tayeb ${ }^{44}$, Mukund Uplekar ${ }^{1}$, Marieke J. van der Werf ${ }^{39}$, \\ Wim Vandevelde ${ }^{45}$. Femke van Kessel ${ }^{36}$, Anna van't Hoog ${ }^{46}$, Jay K. Varma ${ }^{28}$, \\ Natalia Vezhnina ${ }^{47}$, Constantia Voniatis ${ }^{48}$, Marije Vonk Noordegraaf-Schouten ${ }^{36}$, \\ Diana Weil ${ }^{1}$, Karin Weyer ${ }^{1}$, Robert John Wilkinson ${ }^{37,49}$, Takashi Yoshiyama ${ }^{50}$, \\ Jean Pierre Zellweger ${ }^{51}$ and Mario Raviglione ${ }^{1}$
}

ABSTRACT Latent tuberculosis infection (LTBI) is characterised by the presence of immune responses to previously acquired Mycobacterium tuberculosis infection without clinical evidence of active tuberculosis (TB). Here we report evidence-based guidelines from the World Health Organization for a public health approach to the management of LTBI in high risk individuals in countries with high or middle upper income and TB incidence of $<100$ per 100000 per year. The guidelines strongly recommend systematic testing and treatment of LTBI in people living with HIV, adult and child contacts of pulmonary TB cases, patients initiating anti-tumour necrosis factor treatment, patients receiving dialysis, patients preparing for organ or haematological transplantation, and patients with silicosis. In prisoners, healthcare workers, immigrants from high TB burden countries, homeless persons and illicit drug users, systematic testing and treatment of LTBI is conditionally recommended, according to TB epidemiology and resource availability. Either commercial interferon-gamma release assays or Mantoux tuberculin skin testing could be used to test for LTBI. Chest radiography should be performed before LTBI treatment to rule out active TB disease. Recommended treatment regimens for LTBI include: 6 or 9 month isoniazid; 12 week rifapentine plus isoniazid; 3-4 month isoniazid plus rifampicin; or 3-4 month rifampicin alone.

$@$ ERSpublications

Guidelines on LTBI for low TB incidence countries - essential element of the @WHO \#EndTB strategy and TB elimination http://ow.ly/RW8xn 
Affiliations: ${ }^{1}$ The Global TB Programme, World Health Organization, Geneva, Switzerland. ${ }^{2}$ Dept of Infection and Population Health, University College London, London, UK. ${ }^{3}$ Public Health England, London, UK. ${ }^{4}$ World Health Organization, Regional Office for Eastern Mediterranean, Egypt. ${ }^{5}$ National TB Programme, Ministry of Health, Brasilia, Brazil. ${ }^{6}$ Independent consultant, Switzerland. ${ }^{7}$ Pedro Kourí Institute of Tropical Medicine, La Habana, Cuba. ${ }^{8}$ Unit of Infectious Diseases, Dept of Medicine, Karolinska Institute Solna and Karolinska University Hospital, Stockholm, Sweden. ${ }^{9}$ Dept of Pulmonology and Respiratory Medicine, Faculty of Medicine, University of Indonesia and Persahabatan Hospital, Jakarta, Indonesia. ${ }^{10}$ Evandro Chagas National Institute of Infectious Diseases, FIOCRUZ, Rio de Janeiro, Brazil. ${ }^{11}$ Hospital Nacionale Rosales, San Salvador, El Salvador. ${ }^{12}$ Center for TB Research, John Hopkins University, Baltimore, MD, USA. ${ }^{13}$ Tan Tock Seng Hospital, Singapore. ${ }^{14}$ TB Advocacy Consortium, Nairobi, Kenya. ${ }^{15}$ London School of Hygiene and Tropical Medicine, Blantyre, Malawi. ${ }^{16}$ World Health Organization, Regional Office for Europe, Denmark. ${ }^{17}$ Victorian Tuberculosis Program, Melbourne, Australia. ${ }^{18} \mathrm{KNCV}$ Tuberculosis Foundation, Den Haag, The Netherlands. ${ }^{19}$ Dept of HIV and Global Hepatitis Programme, World Health Organization, Switzerland. ${ }^{20}$ Public Health Agency of Canada, Ottawa, ON, Canada. ${ }^{21}$ Istituto Nazionale Malattie Infettive L. Spallanzani, Rome, Italy. ${ }^{22}$ Dept of HIV/AIDS and TB Control Korea, Korea Centers for Disease Control and Prevention, Republic of Korea. ${ }^{23}$ Health Systems Research Unit, South African Medical Research Council, South Africa. ${ }^{24}$ Dept of Clinical Research, London School of Hygiene and Tropical Medicine, London, UK. ${ }^{25}$ Dept of Epidemiology, Boston University School of Public Health, Boston, MA, USA. ${ }^{26}$ Main Medical Department, Ministry of Justice, Azerbaijan. ${ }^{27}$ McGill International TB Centre, and Dept of Epidemiology and Biostatistics, McGill University, Montreal, QC, Canada. ${ }^{28}$ Centers for Disease Control and Prevention, USA. ${ }^{29}$ Dept of Public Health Sciences, Karolinska Institute, Solna, Sweden. ${ }^{30}$ Woolcock Institute of Medical Research University of Sydney and UNSW Australia, Sydney, Australia. ${ }^{31}$ WHO Collaborating Center for TB and Lung Diseases Fondazione S. Maugeri, Tradate, Italy. ${ }^{32}$ Migration Health Department, International Organization of Migration, Geneva, Switzerland. ${ }^{33}$ Infectious Disease Division, Bureau for Global Health, US Agency for International Development, Washington, DC, USA. ${ }^{34}$ Zambart Project, Lusaka, Zambia. ${ }^{35}$ World Health Organization, Regional Office for the Western Pacific, Philippines. ${ }^{36}$ Pallas Health Research and Consultancy BV, Rotterdam, The Netherlands. ${ }^{37}$ Institute of Infectious Diseases and Molecular Medicine, University of Cape Town, Cape Town, South Africa. ${ }^{38}$ Knowledge, Ethics and Research Department, World Health Organization, Switzerland. ${ }^{39}$ European Centre for Disease Prevention and Control, Stockholm, Sweden. ${ }^{40}$ Dept of Clinical Epidemiology and Biostatistics and Dept of Medicine, GRADE Center, McMaster University, Hamilton, ON, Canada. ${ }^{41}$ Dept of Medicine, All India Institute of Medical Sciences, New Delhi, India. ${ }^{42}$ Sassari University, Sassari, Italy. ${ }^{43}$ Vanderbilt University School of Medicine, Nashville, TN, USA. ${ }^{44}$ National TB Programme, Ministry of Health, Riyadh, Saudi Arabia. ${ }^{45}$ European AIDS Treatment Group, Brussels, Belgium. ${ }^{66}$ Academic Medical Centre, University of Amsterdam, Dept of Global Health, Amsterdam, The Netherlands. ${ }^{47}$ HIV/TB and Penal System Projects, Russian Federation. ${ }^{48}$ Clinical Laboratories, Ministry of Health, Nicosia, Cyprus. ${ }^{49}$ Francis Crick Institute Mill Hill Laboratory, Dept of Medicine, Imperial College London, London, UK. ${ }^{50}$ Fukujuji Hospital, Japan Anti Tuberculosis Association, Tokyo, Japan. ${ }^{51}$ Tuberculosis Competence Centre, Berne, Switzerland.

Correspondence: Haileyesus Getahun, Global TB Programme, World Health Organization, 20 Avenue Appia $\mathrm{CH}-1211$ Geneva, Switzerland. E-mail getahunhawho.int

\section{Introduction}

Latent tuberculosis infection (LTBI) is characterised by the presence of immune responses to Mycobacterium tuberculosis infection without clinical evidence of active tuberculosis (TB) [1,2]. One third of the world's population is estimated to be infected with Mycobacterium tuberculosis [3]. The vast majority of infected persons have no signs or symptoms of TB disease and are not infectious, but they are at risk for developing active TB disease and becoming infectious [3]. The lifetime risk of reactivation TB for a person with documented LTBI is estimated to be $5-15 \%$, with the majority developing TB disease within the first 5 years after initial infection [4]. However, the likelihood of progression of LTBI to active TB depends on bacterial, host, and environmental factors [3]. The reactivation of TB can be averted by preventive treatment. Currently available regimens for the treatment of LTBI have an efficacy ranging from $60 \%$ to $90 \%$, the protection of which can last for up to 19 years [5]. The potential benefit of treatment needs to be carefully balanced against the risk of drug-related adverse events. For infected individuals in population groups with a high risk of progression to active disease, the anticipated benefits are usually greater than the potential harms. It is thus important to identify which groups would benefit most.

Guidelines were developed by the World Health Organization (WHO) in response to demand from several member states for clear policy guidance on the management of LTBI. In addition, such guidelines will facilitate in achieving the ambitious targets of the WHO End TB Strategy [6] of a 90\% reduction in TB incidence and a $95 \%$ reduction in $\mathrm{TB}$ deaths by 2035 , and will contribute to the elimination of $\mathrm{TB}$, particularly in low TB incidence settings [7].

The findings and conclusions in this article are those of the authors and do not necessarily represent the views of the US Centers for Disease Control and Prevention or the US Agency for International Development.

Conflict of interest: Disclosures can be found alongside the online version of this article at erj.ersjournals.com

The content of this work is copyright of the authors or their employers. Design and branding are copyright OERS 2015. ERJ Open articles are open access and distributed under the terms of the Creative Commons Attribution NonCommercial Licence 4.0. 
This paper summarises the WHO guidelines on the management of LTBI, which provide guidance for addressing LTBI within a public health approach, and describes recommendations on who should be tested and treated for LTBI, what diagnostic algorithm should be used, as well as which treatment regimens should be adopted, in high and upper-middle income countries with a TB incidence less than 100 per 100000 population per year.

\section{Methods}

The process and procedures for the development of the guidelines complied with the WHO Guidelines Review Committee requirements, including the establishment of a guidelines development panel, a systematic review of the evidence, and formulation of recommendations using a structured process [8]. The overall approach for the management of LTBI requires a comprehensive package of interventions that include: identifying populations at risk; adopting the appropriate diagnostic algorithm; delivering effective and safe treatment in a way that the majority of those who start treatment complete it with no or a minimal risk of adverse events; and developing a system for monitoring and evaluation (figure 1). This package provided the framework for the development of the guidelines. Accordingly, key questions were formulated using the population, intervention, comparator, and outcomes (PICO) format to define the systematic reviews, and their relevant outcomes were selected and rated. From January to May 2014, a total of 14 systematic reviews were undertaken to inform the guidelines development.

The quality of evidence and strength of recommendations were assessed using the Grading of Recommendations Assessment, Development and Evaluation (GRADE) methodology when applicable [9-11]. In the GRADE process, the quality of a body of evidence (high, moderate, low or very low) is defined as the extent to which one can be confident that the reported estimates of effect (desirable or undesirable) available from the evidence are close to the actual effects of interest. The usefulness of an estimate of the effect (of the intervention) depends on the level of confidence in that estimate (i.e. the quality of evidence). The higher the quality of evidence, the more likely a strong recommendation can be made; however, the decision regarding the strength of the recommendation also depends on other factors, including the balance of desirable benefits and undesirable harms, the values and preferences of clients and healthcare providers, as well as resource implications.

In accordance with GRADE, the recommendations in these guidelines were graded into two categories [12-14]. A strong recommendation was one for which the panel was confident that the desirable effects of adherence to the recommendation outweighed the undesirable effects. This could be either in favour of or against an intervention. A conditional recommendation was one for which the panel concluded that the desirable effects of adherence to the recommendation probably outweighed the undesirable effects, but the panel was not confident about these trade-offs. Reasons for not being confident included: absence of high quality evidence (data to support the recommendation were scant); presence of imprecise estimates of benefits or harms (new evidence may result in changing the balance of risk to benefit); uncertainty or variation regarding how different individuals value the outcomes (only applicable to a specific group,

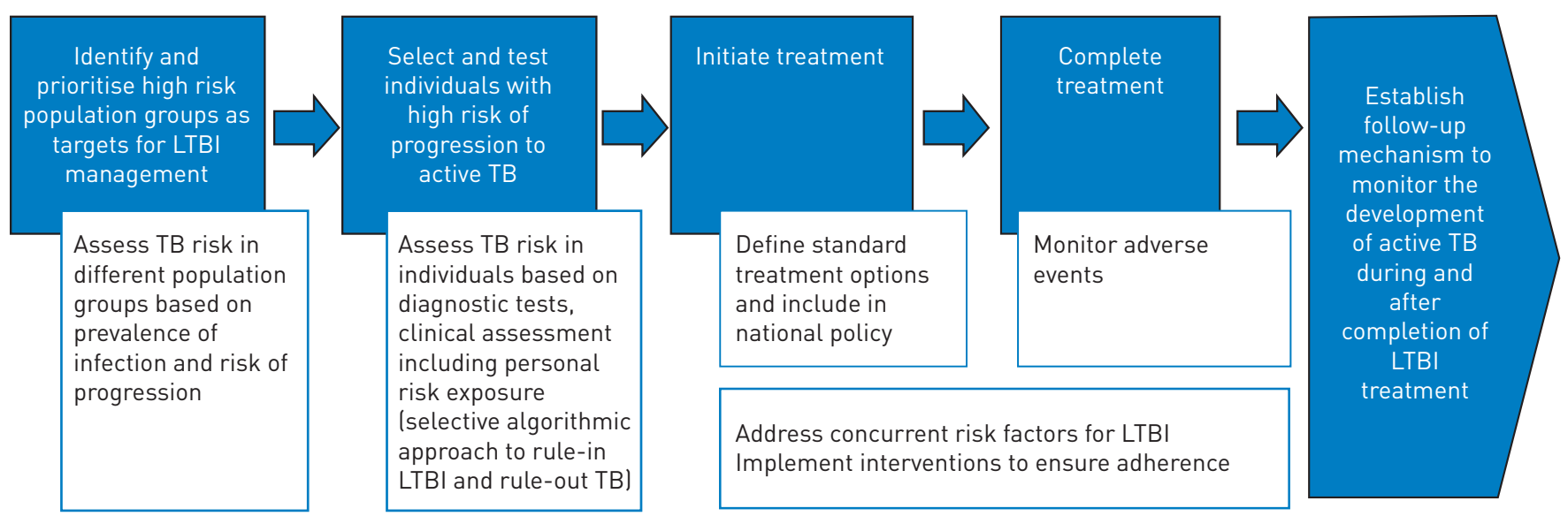

Provide programmatic support: algorithm-based national guidelines targeting high risk population groups; proper documentation reporting and monitoring of people receiving LTBI treatment; functional supply system for diagnostic tests, drugs and other treatments; promote implementation and basic science research to develop service delivery models and scale up novel evidence based interventions.

FIGURE 1 Schematic approach for programmatic management of latent tuberculosis infection (LTBI). TB: tuberculosis. 
population or setting); small benefits and benefits that may not be worth the costs (including the costs of implementing the recommendation) [15].

The desirable effects that were considered included beneficial health outcomes (e.g. prevention and early diagnosis of TB, reduced TB-related morbidity and mortality, less transmission), less burden, and more cost savings; whereas undesirable effects included harms (adverse drug-related events), more burden, and more costs. Burdens considered included the demands of adhering to the recommendations that programmes, patients or caregivers (e.g. family) may have to bear, such as having to undergo more frequent tests, taking additional medications, or opting for a treatment that has a risk for toxicity.

In principle, any country could benefit from systematic management of LTBI. However, these guidelines are primarily targeted to high income or upper middle income countries with an estimated TB incidence rate of less than 100 per 100000 population per year. The panel judged that these countries were most likely to benefit from programmatic management of LTBI due to their current TB epidemiology and resource availability. Therefore, data analysis of some of the systematic reviews considered a composite stratification of countries based on World Bank classification of income [16] and WHO estimate of TB incidence [17].

\section{Results}

Target countries for the guidelines

National TB Programmes or their equivalents in the ministries of health of high income or upper middle income countries with an estimated TB incidence rate of less than 100 per 100000 population per year are the primary target audience for the guidelines presented here (table 1). The guidelines are also relevant for other policy makers responsible for prison or social services, as well as immigration services (such as ministries of justice or correctional services, and ministries dealing with immigration). In addition to the guidelines described in this manuscript there are pertinent WHO guidelines on management of LTBI in people living with HIV [18] and child contacts below 5 years of age [19] which should be implemented in all countries.

\section{Identification of at risk populations}

Management of LTBI can prevent TB which is generated by progression from latent infection to disease. To assist in the identification of groups to prioritise for LTBI testing and treatment, we conducted a primary systematic review of the literature on the relative risk of progression from infection to disease in 24 pre-defined population groups. As evidence was very limited, two additional systematic reviews were conducted to measure the prevalence of LTBI, as well as incidence of active TB, relative to the general population, in those 24 groups.

The first systematic review assessed the risk of progression from LTBI to active TB and was restricted to studies published from January 1, 2003 to January 17, 2014 in English, Spanish, French, and identified in MEDLINE. In addition, expert researchers were also contacted to identify any studies that met the inclusion criteria irrespective of date of publication. Eight individual studies provided evidence of an increased risk of progression from LTBI to active TB for the following risk groups: people living with HIV, adult contacts of TB cases, patients undergoing dialysis, underweight people, individuals with fibrotic radiologic lesions and Mantoux tuberculin skin test (TST) converters within the past 2 years.

The second systematic review assessed the prevalence of Mycobacterium tuberculosis infection as determined either by TST or commercially available interferon-gamma release assays (IGRAs) and was restricted to studies published from January 1, 2003 to January 17, 2014 in English, Spanish, and French, and identified in MEDLINE. A total of 276 studies (with 299 entries) were included. Pooled relative risks were calculated by comparison of LTBI prevalence in risk groups (from individual studies) and the general population (using estimates derived from modelling) [20]. Considerable between-study heterogeneity was observed even after stratification for risk group. Nevertheless, an increased prevalence of LTBI evidenced by positive TST or IGRA, compared to the general population, was reported in at least $65 \%$ of the identified studies for each of the following risk groups: adult and child contacts of persons with TB, prisoners, homeless people, elderly people, immigrants from high TB burden countries, and illicit drug users.

The third systematic review was conducted to determine the pooled incidence rate ratio of active TB in the pre-defined risk groups compared with the general population. The search was restricted to studies published from January 1, 2004 to March 31, 2014 in English and identified through PUBMED. Evidence of increased risk of active TB was reported in the following risk groups: people living with HIV, adult and child contacts of a TB case, patients receiving dialysis, patients receiving anti-tumour necrosis factor (TNF)-alpha drugs, patients with silicosis, healthcare workers (including students), immigrants from high TB burden countries, prisoners, the homeless, persons with cancer, diabetes mellitus, or harmful alcohol use, tobacco smokers and underweight people. 
TABLE 1 Primary target countries for the guidelines on latent tuberculosis infection (LTBI) management ${ }^{\#}$

\begin{tabular}{|c|c|c|c|c|}
\hline EUR & AMR & WPR & EMR & SEAR and AFR \\
\hline Andorra & Argentina & Australia & Iran (Islamic Republic of) & Seychelles \\
\hline Azerbaijan & Bahamas & China & Jordan & Algeria \\
\hline Belarus & Barbados & China, Hong Kong SAR & Kuwait & \\
\hline Belgium & Belize & China, Macao SAR & Lebanon & \\
\hline Croatia & Canada & Guam & Qatar & \\
\hline Cyprus & Cayman Islands & Japan & Saudi Arabia & \\
\hline Czech Republic & Chile & Korea, Republic of & Tunisia & \\
\hline Denmark & Colombia & Malaysia & United Arab Emirates & \\
\hline Estonia & Costa Rica & New Caledonia & & \\
\hline Finland & Cuba & New Zealand & & \\
\hline Ireland & Jamaica & & & \\
\hline Israel & Mexico & & & \\
\hline Italy & Panama & & & \\
\hline Latvia & Puerto Rico & & & \\
\hline Lithuania & St. Kitts and Nevis & & & \\
\hline Luxembourg & Saint Lucia & & & \\
\hline Malta & Saint Vincent and & & & \\
\hline Monaco & the Grenadines & & & \\
\hline Montenegro & Sint Maarten & & & \\
\hline The Netherlands & Suriname & & & \\
\hline Norway & Trinidad and Tobago & & & \\
\hline Poland & Turks and Caicos Islands & & & \\
\hline Sweden & & & & \\
\hline Switzerland & & & & \\
\hline The former Yugoslav & & & & \\
\hline Republic of Macedonia & & & & \\
\hline Turkey & & & & \\
\hline Turkmenistan & & & & \\
\hline UK & & & & \\
\hline
\end{tabular}

EUR: region of Europe; AMR: region for the Americas; WPR: region for Western Pacific; EMR: Eastern Mediterranean region; SEAR: South-East Asia region; AFR: Africa region. "\# : high income and upper middle income countries according to World Bank income classification (2013) with annual tuberculosis incidence of less than 100 cases per 100000 population (2013).

Based on these findings the panel issued the following recommendations (box 1):

- Systematic testing and treatment of LTBI should be performed in people living with HIV, adult and child contacts of pulmonary TB cases, persons initiating anti-TNF-alpha treatment, receiving dialysis, preparing for organ or haematological transplantation, or with silicosis. Either IGRA or Mantoux TST should be used to test for LTBI. (Strong recommendation, low to very low quality of evidence.)

- Systematic testing and treatment of LTBI should be considered for prisoners, healthcare workers, immigrants from high TB burden countries, homeless persons and illicit drug users. Either IGRA or Mantoux TST should be used to test for LTBI. (Conditional recommendation, low to very low quality of evidence.) 
- Systematic testing for LTBI is not recommended in people with diabetes, people with harmful alcohol use, tobacco smokers, and underweight people unless they are already included in the above recommendations. (Conditional recommendation, very low quality of evidence.)

The rationale for the panel to make strong recommendations despite low to very low quality of evidence was based on the increased likelihood of progression to active TB disease, and that the benefits of treatment outweighed the potential harms in those at-risk population groups. Conditional recommendations were made primarily because of reservations on implementation issues, as well as low quality of the evidence.

There was a paucity of data on the benefits and harms of systematic LTBI testing and treatment as well as doubts about its operational feasibility in diabetic patients, people with harmful alcohol use, tobacco smokers and underweight people.

\section{Excluding active TB and testing for LTBI}

Exclusion of active TB disease is an important initial step in the process of LTBI management. The fourth systematic review had been conducted in 2012 to determine the accuracy of symptoms and chest radiography screening for active pulmonary TB in HIV-negative persons and persons with unknown HIV status [21] as a screening rule for people living with HIV already exists [22]. The databases MEDLINE, EMBASE, LILIACS and Health Technology Assessment were searched from 1992 to 2012, supplemented by a search of reference lists of relevant reviews and studies, websites of the WHO, and expert consultation for relevant studies and unpublished reports.

The review identified 11 studies from general population surveys that provided data on screening with either symptoms or chest radiography or both [21]. Based on the review, a model was constructed to compare seven screening strategies to rule out active TB (table 2). The presence of any symptom suggestive of TB (i.e. any one of cough, haemoptysis, fever, night sweats, weight loss, chest pain, shortness of breath and fatigue) plus any abnormality on chest radiography offered the highest sensitivity and negative predictive value to rule out active TB.

The fifth systematic review explored tests and clinical proxies that can best identify individuals most at risk of progression to incident TB disease. While the systematic review did not identify any clinical parameter that would assist in the prediction of progression to active TB disease, 29 studies that provided information on the utility of IGRAs and TST in predicting the risk of TB were assessed. The main effect measure of interest was the risk ratio, comparing $\mathrm{TB}$ cumulative incidence following a positive test result versus a negative test result in individuals not receiving preventive therapy, or the incidence rate ratio in the few studies that reported the person-years of follow-up amongst persons with positive and negative tests.

BOX 1 Recommendations of the guidelines in high and upper-middle income countries with a tuberculosis (TB) incidence less than 100 per 100000 population per year

- Systematic testing and treatment of latent tuberculosis infection (LTBI) should be performed in people living with HIV, adult and child contacts of pulmonary TB cases, patients initiating anti-tumour necrosis factor treatment, patients receiving dialysis, patients preparing for organ or haematological transplantation, and patients with silicosis. Either interferon-gamma release assays (IGRA) or Mantoux tuberculin skin test (TST) should be used to test for LTBI. (Strong recommendation, low to very low quality of evidence.)

- Systematic testing and treatment of LTBI should be considered for prisoners, health-care workers, immigrants from high TB burden countries, homeless persons and illicit drug users. Either IGRA or TST should be used to test for LTBI. (Conditional recommendation, low to very low quality of evidence.)

- Systematic testing for LTBI is not recommended in people with diabetes, people with harmful alcohol use, tobacco smokers, and underweight people provided they are not already included in the above recommendations. (Conditional recommendation, very low quality of evidence.)

- Individuals should be asked about symptoms of TB before being tested for LTBI. Chest radiography can be performed if efforts are intended also for active TB case finding. Individuals with TB symptoms or any radiological abnormality should be investigated further for active TB and other conditions. (Strong recommendation, low quality of evidence.)

- Either TST or IGRA can be used to test for LTBI in high-income and upper middle-income countries with estimated TB incidence less than 100 per 100000 . (Strong recommendation, low quality of evidence.) IGRA should not replace TST in low-income and other middle-income countries. (Strong recommendation, very low quality of evidence.)

- Treatment options recommended for LTBI include: 6-month daily isoniazid, or 9 month daily isoniazid, or 3 month weekly rifapentine plus isoniazid, or 3-4 month daily isoniazid plus rifampicin, or 3-4 month daily rifampicin alone. (Strong recommendation, moderate to high quality of evidence.) 
TABLE 2 Performance of different screening strategies to rule out active tuberculosis (TB) before latent TB infection treatment based on a hypothetical cohort population of 1000 at a baseline TB prevalence of $0.5 \%$ [21]

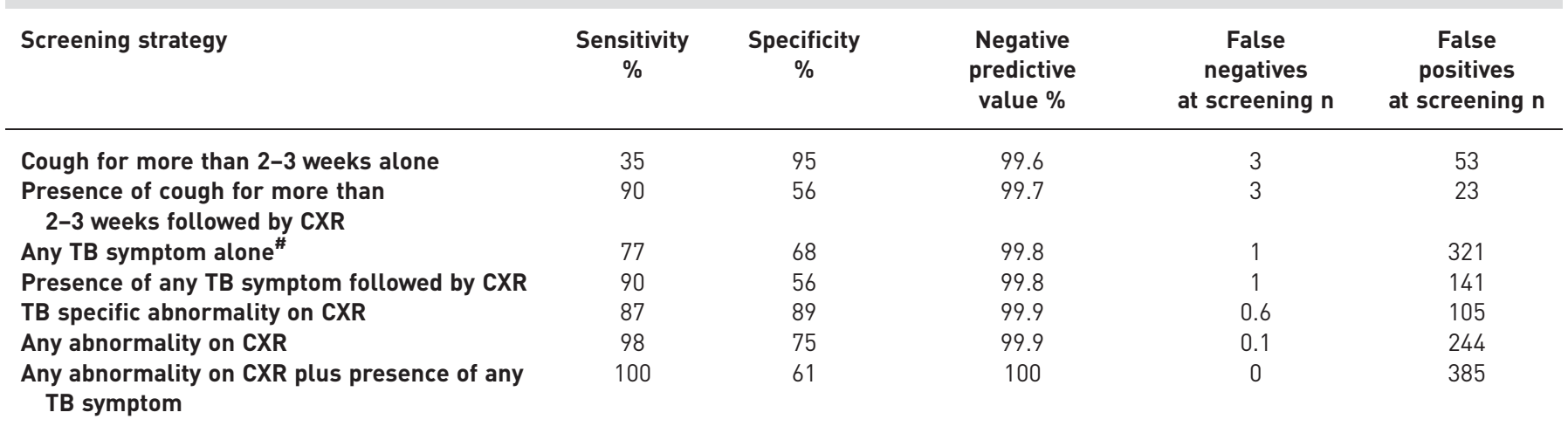

CXR: chest radiograph. ${ }^{\#}$ : includes any one of: cough, haemoptysis, fever, night sweats, weight loss, chest pain, shortness of breath and fatigue.

The main analysis was restricted to the eight studies that compared TST and IGRA to each other in the same study population (head-to-head comparison). The overall pooled risk ratio for test positives compared to test negatives for TST was 2.58 (95\% CI 1.72-3.88) and for IGRA 4.94 (95\% CI 1.79-13.65). Similarly, the pooled incidence rate ratio in the three head-to-head studies with person-time data was 2.07 (95\% CI 1.38-3.11) for TST and 2.40 (95\% CI 1.26-4.60) for IGRA. In both analyses, the confidence intervals around the effect measures for the TST and IGRA were imprecise and largely overlapped. There was insufficient data to provide evidence on predictive utility of the tests among specific high risk groups. The added value of the tests over and above other clinical indicators of risk as well as their additive value when combining TST and IGRAs for diagnosis of LTBI could not be evaluated.

Based on these findings the panel issued the following recommendations:

- Individuals should be asked about symptoms of TB before being tested for LTBI. Chest radiography can be performed if efforts are intended also for active TB case finding. Individuals with TB symptoms or any radiological abnormality should be investigated further for active TB and other conditions. (Strong recommendation, very low quality of evidence.)

- Either TST or IGRA can be used to test for LTBI in high income and upper middle income countries with an estimated TB incidence of less than 100 per 100000 . (Strong recommendation, very low quality of evidence.)

Furthermore, the panel maintained the existing WHO recommendation that IGRA should not replace TST in low income and other middle income countries (strong recommendation, very low quality of evidence) [23].

\section{Treatment options for LTBI}

The sixth systematic review was conducted to evaluate the efficacy and safety of treatment for LTBI [24]. 53 studies, all of which were randomised controlled trials and recorded at least one of the two pre-specified end-points (preventing active TB, hepatotoxicity of grade III or above), were included. Data was available for 15 treatment regimens, although relatively few direct comparisons were reported. In addition to standard meta-analysis, mixed treatment comparisons were also performed to allow the inference of indirect evidence (regimen comparisons without randomised controlled trials) and thus a network of evidence [24].

The efficacy of several regimens was established in trials against placebo or no treatment, including 6 month and 12 month isoniazid, 4 month rifampicin, and 3 month rifampicin and isoniazid. No trial determined the efficacy and safety of a 3 month regimen of weekly rifapentine plus isoniazid compared to placebo or no treatment, because this regimen was introduced at a time when conducting placebo controlled trials for LTBI was not considered ethically acceptable.

The main analysis considered studies that compared the above regimens against 6 month isoniazid (taken as reference) for efficacy and heptotoxicity (table 3). In general, these comparisons did not show superiority of efficacy of one regimen over any other. However, in terms of safety, a 3-4 month rifampicin regimen and a 3 month weekly rifapentine plus isoniazid regimen had fewer hepatotoxicity events compared to the 6 month and 9 month isoniazid regimen, respectively. In the absence of any direct 
TABLE 3 Standard random effects meta-analysis comparison of efficacy and hepatotoxicity among various treatment regimens for treatment of latent tuberculosis (TB) infection

\begin{tabular}{llrl} 
Comparator & Intervention & Development of incident TB & Hepatotoxicity \\
\hline Placebo & Isoniazid 6 months & $0.61(0.48-0.77)$ & $0.99(0.42-2.32)$ \\
Placebo & Isoniazid 12-72 months & $0.53(0.41-0.69)$ & $0.59(0.23-1.55)$ \\
Placebo & Rifampicin 3-4 months & $0.48(0.26-0.87)$ & \\
Placebo & Rifampicin and isoniazid 3-4 months & $0.52(0.33-0.84)$ & \\
Isoniazid 6 month & Rifampicin 3-4 months & $0.78(0.41-1.46)$ & $0.89(0.65-1.23)$ \\
Isoniazid 6 month & Rifampicin and isoniazid 3-4 months & $1.09(0.60-1.99)$ & $0.89(0.52-1.55)$ \\
Isoniazid 6 month & 3 month weekly rifapentine plus isoniazid & $0.00-0.48)$ \\
Isoniazid 9 month & 3 month weekly rifapentine plus isoniazid & $0.44(0.18-1.07)$ & $0.16(0.10-0.27)$ \\
\hline
\end{tabular}

Data are presented as odds ratios with $95 \%$ confidence intervals. ${ }^{\#}$ : exclusively among people living with HIV.

comparison of efficacy of 6 and 9 month isoniazid, the equivalence of these two regimens was based on re-analysis of the US Public Health Service (USPHS) trials conducted in the 1950s and 1960s that concluded that optimal protection from isoniazid appears to be obtained by 9 months [25]. Pyrazinamide-containing regimens were evaluated in the analysis, but were not considered for recommendation because of high documented toxicity $[3,26]$.

Based on these findings the following treatment options were recommended for the treatment of LTBI: 6 month isoniazid, or 9 month isoniazid, or 3 month regimen of weekly rifapentine plus isoniazid, or 34 months isoniazid plus rifampicin, or 3-4 months rifampicin alone (strong recommendation, moderate to high quality of evidence).

\section{Preventive treatment for contacts of multidrug resistant-TB cases}

The seventh systematic review was conducted to define the effectiveness of anti-TB drugs in preventing active TB in contacts of multidrug-resistant (MDR)-TB patients. Four studies were included for the analysis; all were cohort studies of which one [27] was a prospective study exclusively involving children below 5 years of age while the others were retrospective studies involving both adults and children [28-30]. For the final analysis, one study was excluded for its small sample size [29] while two other studies were excluded because all or the majority of MDR-TB contacts received preventive treatment with isoniazid [28, 30]. Therefore, the quality of evidence was determined using only one comparison study which used a tailored treatment regimen taking into account the resistance pattern of the index case among childhood contacts [27]. In this single study two of 41 children receiving tailored preventive therapy developed TB (confirmed and probable TB) compared to 13 of 64 children not receiving preventive treatment (OR $0.2,95 \%$ CI $0.04-0.94)$.

There is thus sparse evidence on the effectiveness and safety of using anti-TB drugs to prevent active TB among adult and childhood contacts of MDR-TB cases. Furthermore, determination of the drug susceptibility profile for drugs to be used as preventive treatment for MDR contacts poses both technical and logistic challenges and may lead to drug-related harms, which would necessitate additional cost for close monitoring which has resource implications [31]. The panel noted the serious limitations of evidence to draw any recommendations on MDR-TB preventive therapy as a public health measure and concluded that the management of contacts of MDR-TB patients needs to be guided by a comprehensive clinical risk assessment considering the balance between risk and benefits for the individual. Based on the available evidence and the probability of increased likelihood to develop active TB disease following recent infection, strict clinical observation and close monitoring for the development of active TB disease for at least 2 years is preferred over the provision of preventive treatment for contacts of MDR-TB cases.

\section{Adverse event monitoring}

Drug-specific adverse reactions can occur with individuals who receive treatment for LTBI who are usually in good condition and not sick, making it more important to minimise risks of drug-induced harms during treatment. Adverse drug reactions in persons who take isoniazid include asymptomatic elevation of serum liver enzyme concentrations, peripheral neuropathy and hepatotoxicity. Rifampicin- and rifapentine-related adverse reactions include cutaneous reactions, hypersensitivity or flu-like reactions, gastrointestinal intolerance and hepatotoxicity. While most adverse drug reactions are minor and occur rarely, particular attention should be paid to prevent drug-induced hepatotoxicity. 
The eighth systematic review was conducted to assess the best way to monitor and manage hepatotoxicity and other adverse drug reactions and no studies were identified [32]. A review of national guidelines [33-37] instead showed consistent recommendations based on expert opinion, which were useful to inform the judgment of the panel.

Based on these observations, the panel underlined the importance of routine regular clinical monitoring of individuals receiving treatment for LTBI through a monthly visit to healthcare providers. The prescribing healthcare provider should explain the disease process and the rationale of treatment and emphasise the importance of completing it. Those receiving treatment should be educated to contact their healthcare providers should they develop symptoms such as anorexia, paraesthesiae, nausea, vomiting, abdominal discomfort, persistent fatigue or weakness, dark-coloured urine, pale stools or jaundice. Whenever a healthcare provider cannot be consulted at the onset of these symptoms, treatment should be stopped immediately.

The panel noted that there was insufficient evidence to support baseline laboratory testing for measurements of serum aspartate aminotransferase, alanine aminotransferase and bilirubin. However, the panel strongly encouraged baseline laboratory testing for individuals with any of the following risk factors: history of liver disease; regular use of alcohol; chronic liver disease; HIV infection; age more than 35 years; and pregnancy or the immediate postpartum period (i.e., within 3 months of delivery). For individuals with abnormal baseline test results, routine periodic laboratory testing should be carried out.

\section{Risk of drug resistance following LTBI treatment}

The ninth systematic review was conducted to determine whether LTBI treatment leads to significant development of resistance. The systematic review considered the following treatment regimens.

\section{Isoniazid for 6-12 month duration}

An update of a previous systematic review was done [38]. 13 studies comparing 6-12 month isoniazid preventive therapy versus no treatment or placebo were included in the systematic review (seven involving HIV uninfected populations). There was no difference in the incidence risk of isoniazid-resistant TB (risk ratio $1.45,95 \%$ CI $0.85-2.47)$. There was little evidence of heterogeneity $(p=0.923)$ and the risk ratio for HIV-uninfected and HIV-infected populations were comparable.

\section{BOX 2 Priority research gaps identified for revision of the guidelines}

- Measurement of the risk of progression from latent tuberculosis infection (LTBI)to active disease in a number of risk groups

- Direct measurement of tuberculosis (TB) incidence in cohort studies of risk groups compared to general population (e.g. tuberculin skin test (TST) positive cohort in a risk group versus TST positive cohort in the general population) or through alternative methods (such as the use of genotyping to measure the risk of reactivation in comparison studies).

- Generating evidence on the benefits and harms of systematic treatment of LTBI in all risk groups and particularly the following groups: diabetic patients, people with harmful alcohol use, tobacco smokers, underweight people, persons with silica exposure, patients receiving steroid treatment, patients with rheumatologic conditions, indigenous populations and cancer patients.

- Social adverse events of LTBI treatment by risk group treated.

- Operational and clinical studies to identify undiagnosed active TB before LTBI treatment initiation

- Diagnostic performance of the algorithm proposed in these guidelines as well as development and evaluation of new algorithms tailored to the needs of specific risk groups.

- Prospective, randomised studies tailored to specific risk group to measure the incremental benefits of routine monitoring of liver enzymes over education and clinical observation alone in terms of preventing severe clinical adverse events.

- Programme-based surveillance systems and clinical studies to monitor the risk of drug resistance following LTBI treatment.

- Efficacy studies of currently recommended treatment options in areas of highly prevalent drug resistance

- The effectiveness of context-specific interventions to enhance adherence and treatment completion tailored by risk groups.

- Direct measurement of cost-effectiveness in specific settings and risk populations.

- Adequately powered randomised controlled trials to define the benefits and harms of treatment of multidrug resistant TB contacts.

- Epidemiological studies to understand the burden of LTBI and inform the development of nationally and locally tailored interventions. 
Rifamycin-containing regimens

Five studies were included in the comparison of rifamycin resistance in individuals treated with a rifamycin-containing regimen versus a regimen not containing rifamycin. There were very few cases of rifamycin resistance, a total of six $(0.1 \%)$ cases in 5790 individuals receiving LTBI treatment with a rifamycin and five $(0.09 \%)$ cases in the 5537 individuals in the control group with a relative risk of 1.12 (95\% CI 0.41-3.08). The quality of the evidence was very low.

The panel concluded that the available evidence showed no significant association between anti-TB drug resistance and the use of isoniazid and/or rifamycins for LTBI. However, the panel emphasised the importance of establishing national TB drug resistance surveillance systems in countries implementing systematic testing and treatment for LTBI.

\section{Adherence and completion of preventive treatment}

Adherence to the full course and completion of LTBI treatment are important determinants of clinical benefit to the individual as well as to the success of the programme.

Four systematic reviews (10-13th) were conducted to describe initiation and completion rates of LTBI treatment; to define determinants of initiation, adherence and completion rates of LTBI treatment; to assess interventions that are effective to improve those rates; and to assess if the duration of LTBI treatment will be a barrier to implementation of LTBI treatment. All four reviews focused on prospective studies.

Completion rates were shown to vary greatly across risk groups ranging from $6 \%$ to $94 \%$. In general, completion rates were lower among prisoners and immigrants compared with people living with HIV and contacts, and were inversely proportional related to the duration of treatment.

Determinants of treatment initiation, adherence and completion identified in the systematic review were: 1) adverse drug reactions, 2) longer duration of treatment, 3) legal status among immigrants, 4) long distance from health facility, 5) history of incarceration, 6) absence of perception of risk, 7) presence of stigma, 8) alcohol and drug use, 9) unemployment, and 10) time lag between diagnosis and treatment.

Evidence on the efficacy of interventions to improve treatment adherence and completion showed that shorter treatment duration was significantly associated with increased adherence [39-41]. There is contradictory evidence on the role of monetary incentives to improve treatment completion rates, with randomised trials showing benefit of incentives (either monetary or methadone) on treatment completion rates among illicit drug users [42, 43], and other randomised trials among the homeless [44] and prisoners [45] not showing any significant impact. Significant increases in completion rates were demonstrated with peer support and coaching among adolescents and adults [46-48]; nurse case management among the homeless [49]; cultural case management among immigrants [50]; and educational interventions among inmates [45]. No studies were found on the question whether the duration of protection from LTBI treatment will be a barrier to implementation of LTBI treatment.

It was noted that the available evidence is heterogeneous and inconclusive to be able to recommend the best interventions to improve treatment adherence and completion. However, the panel underlined the importance of designing flexible interventions that are tailored to respond to the local context and needs of the population to ensure acceptable initiation of, adherence to and completion of LTBI treatment as part of the public health approach.

\section{Cost-effectiveness}

The 14th systematic review was conducted to critically appraise and summarise current evidence on the cost-benefit and cost-effectiveness associated with screening for and treatment of LTBI. Studies that evaluated costs and outcomes of any screening strategy and any drug regimen for LTBI compared to no intervention in any setting and population group were selected. The outcomes considered were incremental cost per quality-adjusted life year or life year gained, and incremental cost per TB case averted. 39 articles were included and the majority of articles (82\%) reported on analyses conducted in upper middle-income countries with TB incidence less than 100 per 100000 population.

Cost inputs (adjusted for currency and inflation to US\$ value as of 2012), varied widely among studies. For example, the cost of testing for LTBI using TST varied from US\$ 10.9 in a study from Italy [51] to an average of US\$ 31.5 in studies from the UK [52]; similarly, detecting LTBI using an IGRA test varied from US\$ 22.5 in a study from Mexico [53] to an average of US\$ 97.1 in studies from the UK [52, 54]. Wide variations were also observed for the cost of screening eligible candidates for latent TB treatment and the overall cost. For example, the costs of monitoring adverse drug reactions (including liver function tests and clinical monitoring) ranged from US\$ 8.3 [55] to US\$ 687.3 [56]. The average cost of treating LTBI (including cost of drugs and monitoring) ranged from US\$ 381.9 in Italy [51] to US\$ 1129.9 in the UK [52]. 
Studies showed that LTBI testing and treating of immigrants from high (between 120 and 150 per 100000 population) to low TB incidence countries may result in savings for the healthcare system or have a favourable incremental cost-effectiveness ratio [52, 54, 57-61]. Similar results were found in studies among people living with HIV $[51,55,60,62]$ and contacts of patients with active TB [52, 61, 63-66]. However, a marked variability across studies in the economic inputs, in epidemiological and TB natural history parameters, as well as in assumptions on the effectiveness of preventive treatment made the extrapolation of the results from one setting to another problematic.

\section{Discussion}

The main principle guiding testing and treatment for LTBI is that benefit outweighs the risk to the individual. The decision of national TB programmes and other stakeholders about the priority risk groups for programmatic management of LTBI needs to consider the local epidemiology of TB, and availability and efficient use of resources. Cost-effectiveness analyses based on rigorous empirical data are scarce. However, prioritising high risk groups, such as people living with HIV, adult and child contacts of pulmonary TB cases, as well as immigrants from high to low TB burden countries, for latent TB testing and treatment has the potential to yield savings for the healthcare system. The value of earlier antiretroviral therapy for the prevention of TB among people living with HIV is enormous [67]. Therefore, HIV testing should be incorporated into the clinical evaluation of LTBI treatment candidates based on national policies and local epidemiology of HIV.

The comparative analysis between TST and IGRA in the head-to-head studies showed no evidence that one test should be preferred over the other to assess progression to TB disease. Given the low positive predictive values for TB disease progression for both TST and IGRAs new diagnostic methods need to be developed and evaluated. The resource requirements for choosing the test could vary and the decision needs to consider several factors, including the structure of the health system, feasibility of implementation, infrastructure requirements and bacillus Calmette-Guérin vaccination coverage and practice.

The panel agreed on the equivalence of 6 month isoniazid, 9 month isoniazid, 3 month weekly rifapentine plus isoniazid, 3-4 month isoniazid plus rifampicin and 3-4 month rifampicin alone. However, the panel agreed that shorter duration regimens are preferred over longer duration regimens from the perspective of individuals receiving treatment, clinicians providing the treatment, and programme managers, and concluded that the 3 month regimen of weekly rifapentine plus isoniazid may have possible advantages over the other regimens. Similarly, the panel agreed that 6 month isoniazid is preferred over 9 month isoniazid due to resource requirements, feasibility and acceptability by patients. The panel noted the reported positive acceptability of rifampicin- and rifapentine-containing regimens by individuals receiving treatment, and further concluded that rifampicin (3-4 months isoniazid plus rifampicin and 4-month rifampicin only) and isoniazid (6 and 9 month) containing regimens could be self-administered by individuals receiving treatment. Rifampicin- and rifapentine-containing regimens should be prescribed with caution to people living with HIV who are on antiretroviral treatment due to potential drug-to-drug interactions. National programmes need to decide upon the treatment options to be offered in their country, taking into consideration their resource capacity and national and local context.

In addition to the general ethical considerations in TB programmes [68], LTBI testing and treatment raises a range of ethical issues [69] that need to be addressed through appropriate national policies and practices. Policies should be evaluated under an ethical perspective after implementation, both to consider possible unexpected impact and to ensure that the evidence on which they are based remains current and relevant [70].

The introduction of management of LTBI as a public health intervention will require the documentation of treated individuals through functional and routine monitoring and evaluation systems that are aligned with general national systems. Appropriate recording and reporting tools need to be developed, and standardised indicators established to regularly inform decision making. In some instances, these may require changes in the national legal and policy framework that have to be addressed according to the local and national context. Key indicators to be considered for the monitoring and evaluation system include: coverage of LTBI testing; treatment initiation and completion; the development of active TB during and after the completion of treatment for latent $\mathrm{TB}$; monitoring of drug resistance, as well as reasons for not initiating and completing LTBI treatment. Additionally, programme monitoring is needed to evaluate quality, programme effectiveness and impact. Nationally standardised indicators and data capturing mechanisms using electronic system and digital technology are also required.

National TB programmes or their equivalents need to pursue multi-sectoral engagement and create a conducive policy and programmatic environment, including the development of national and local policies and standard operating procedures to facilitate the implementation of the recommendations in these guidelines. This could include promoting universal health coverage and prioritising the risk groups based 
on the epidemiology of $\mathrm{TB}$, as well as establishing robust health infrastructure and programmatic management. The planning of programmatic LTBI management should be integrated with planning of systematic screening for active TB in high risk groups [71]. Dedicated resources will need to be allocated, including for human resource development and service delivery.

The review of the evidence for formulating these recommendations exposed a number of gaps to better understand, diagnose and treat LTBI (box 2). It is imperative that donors and the scientific research community respond to these gaps in order to update the guidelines and optimise programme implementation. Such research should involve innovative synergies between the public and private sectors in order to overcome market shortcomings [3].

\section{Acknowledgements}

We would like to acknowledge the invaluable comments of all the peer reviewers of the WHO guidelines on the Management of latent tuberculosis. We would like to thank Rosella Centis and Lia D'Ambrosio for their support during the submission of this manuscript.

\section{References}

1 World Health Organization. Guidelines on the Management of Latent Tuberculosis Infection. Geneva, World Health Organization, 2015.

2 Mack U, Migliori GB, Sester M, et al. LTBI: latent tuberculosis infection or lasting immune responses to M. tuberculosis? A TBNET consensus statement. Eur Respir J 2009; 33: 956-973.

3 Getahun H, Matteelli A, Chaisson RE, et al. Latent Mycobacterium tuberculosis infection. N Engl J Med 2015; 372: 2127-2135.

4 Comstock GW, Livesay VT, Woolpert SF. The prognosis of a positive tuberculin reaction in childhood and adolescence. Am J Epidemiol 1974; 99: 131-138.

Lobue P, Menzies D. Treatment of latent tuberculosis infection: an update. Respirology 2010; 15: 603-622.

Uplekar M, Weil D, Lonnroth K, et al. WHO’s new End TB Strategy. Lancet 2015; 385: 1799-1801.

Lonnroth K, Migliori GB, Abubakar I, et al. Towards tuberculosis elimination: an action framework for low-incidence countries. Eur Respir J 2015; 45: 928-952.

8 World Health Organization. WHO Handbook for Guideline Development. 2nd Edn. Geneva, World Health Organization, 2014

9 Guyatt GH, Oxman AD, Vist GE, et al. Rating quality of evidence and strength of recommendations: GRADE: an emerging consensus on rating quality of evidence and strength of recommendations. BMJ 2008; 336: 924.

10 Guyatt G, Oxman AD, Akl E, et al. GRADE guidelines 1. Introduction-GRADE evidence profiles and summary of findings tables. J Clin Epidemiol 2011; 64: 383-394.

11 Guyatt GH, Oxman AD, Schunemann HJ, et al. GRADE guidelines: a new series of articles in the Journal of Clinical Epidemiology. J Clin Epidemiol 2011; 64: 380-382.

12 Andrews J, Guyatt G, Oxman AD, et al. GRADE guidelines: 14. Going from evidence to recommendations: the significance and presentation of recommendations. J Clin Epidemiol 2013; 66: 719-725.

13 Andrews JC, Schunemann HJ, Oxman AD, et al. GRADE guidelines: 15. Going from evidence to recommendation-determinants of a recommendation's direction and strength J Clin Epidemiol 2013; 66: 726-735.

14 Schunemann HJ, Oxman AD, Akl EA, et al. Moving from evidence to developing recommendations in guidelines: article 11 in Integrating and coordinating efforts in COPD guideline development. An official ATS/ERS workshop report. Proc Am Thorac Soc 2012; 9: 282-292.

15 Brunetti M, Shemilt I, Pregno S, et al. GRADE guidelines: 10. Considering resource use and rating the quality of economic evidence J Clin Epidemiol 2013; 66: 140-150.

16 World Bank. Income Classification. http://data.worldbank.org/about/country-and-lending-groups Date last accessed: March 19, 2015.

17 World Health Organization. Global Tuberculosis Control: 2014 Report. Geneva, World Health Organization, 2014.

18 World Health Organization. Guidelines for Intensified Tuberculosis Case-finding and Isoniazid Preventive Therapy for People Living with HIV in Resource-constrained Settings. Geneva, World Health Organization, 2011.

19 World Health Organization. Recommendations for Investigating Contacts of Persons with Infectious Tuberculosis in Low- and Middle-income Countries. Geneva, World Health Organization, 2012.

20 Dye C, Scheele S, Dolin P, et al. Consensus statement. Global burden of tuberculosis: estimated incidence, prevalence, and mortality by country. WHO Global Surveillance and Monitoring Project JAMA 1999; 282: $677-686$.

21 van't Hoog AH, Langendam MW, Mitchell E, et al. A systematic review of the sensitivity and specificity of symptom- and chest-radiography screening for active pulmonary tuberculosis in HIV-negative persons and persons with unknown HIV status. Report to WHO, 2013. www.who.int/tb/Review2Accuracyofscreeningtests.pdf? ua $=1$ Date last accessed: July 7, 2015. 2013.

22 Getahun H, Kittikraisak W, Heilig CM, et al. Development of a standardized screening rule for tuberculosis in people living with HIV in resource-constrained settings: individual participant data meta-analysis of observational studies. PLoS Med 2011; 8: e1000391.

23 World Health Organization. Use of Tuberculosis Interferon-gamma Release Assays (IGRAs) in Low- and Middle-income Countries. Policy Statement. Geneva, World Health Organization, 2011.

24 Stagg HR, Zenner D, Harris RJ, et al. Treatment of latent tuberculosis infection: a network meta-analysis. Ann Intern Med 2014; 161: 419-428.

25 Comstock GW. How much isoniazid is needed for prevention of tuberculosis among immunocompetent adults? Int J Tuberc Lung Dis 1999; 3: 847-850.

26 McElroy PD, Ijaz K, Lambert LA, et al. National survey to measure rates of liver injury, hospitalization, and death associated with rifampin and pyrazinamide for latent tuberculosis infection. Clin Infect Dis 2005; 41: 1125-1133. 
27 Schaaf HS, Gie RP, Kennedy M, et al. Evaluation of young children in contact with adult multidrug-resistant pulmonary tuberculosis: a 30-month follow-up. Pediatrics 2002; 109: 765-771.

28 Attamna A, Chemtob D, Attamna S, et al. Risk of tuberculosis in close contacts of patients with multidrug resistant tuberculosis: a nationwide cohort. Thorax 2009; 64: 271.

29 Denholm JT, Leslie DE, Jenkin GA, et al. Long-term follow-up of contacts exposed to multidrug-resistant tuberculosis in Victoria, Australia, 1995-2010. Int J Tuberc Lung Dis 2012; 16: 1320-1325.

30 Kritski AL, Marques MJ, Rabahi MF, et al. Transmission of tuberculosis to close contacts of patients with multidrug-resistant tuberculosis. Am J Respir Crit Care Med 1996; 153: 331-335.

31 World Health Organization. A Practical Handbook on the Pharmacovigilance of Medicines Used in the Treatment of Tuberculosis: Enhancing the Safety of the TB Patient. Geneva, World Health Organization, 2012.

32 Sotgiu G, Matteelli A, Getahun $\mathrm{H}$, et al. Monitoring toxicity in individuals receiving treatment for latent tuberculosis infection: a systematic review versus expert opinion. Eur Respir J 2015; 45: 1170-1173.

33 Public Health Agency of Canada. Canadian Tuberculosis Standards. 7th Edn. Canadian Thoracic Society and The Public Health Agency of Canada, 2014.

34 Centers for Disease Control and Prevention. Latent Tuberculosis Infection: a Guide for Primary Health Care Providers. Atlanta, US Department of Health and Human Services, Centers for Disease Control and Prevention, National Center for HIV/AIDS, Viral Hepatitis, STD, and TB Prevention, Division of Tuberculosis Elimination, 2013.

35 Ministry of Health (Sweden). Tuberkulos Vägledning för sjukvårdspersonal. Stockholm, Ministry of Health, 2009. www.socialstyrelsen.se/smittskydd/sjukdomar/smittsammasjukdomarochsmittamnen/tuberkulos. Date last accessed: June 25, 2014. 2009.

36 NICE. Tuberculosis - Clinical Diagnosis and Management of Tuberculosis, and Measures for its Prevention and Control. Manchester, National Institute for Health and Clinical Excellence, 2011.

37 Schaberg T, Bauer T, Castell S, et al. [Recommendations for therapy, chemoprevention and chemoprophylaxis of tuberculosis in adults and children. German Central Committee against Tuberculosis (DZK), German Respiratory Society (DGP)]. Pneumologie 2012; 66: 133-171.

38 Balcells ME, Thomas SL, Godfrey-Faussett $\mathrm{P}$, et al. Isoniazid preventive therapy and risk for resistant tuberculosis. Emerg Infect Dis 2006; 12: 744-751.

39 Spyridis NP, Spyridis PG, Gelesme A, et al. The effectiveness of a 9-month regimen of isoniazid alone versus 3 -and 4-month regimens of isoniazid plus rifampin for treatment of latent tuberculosis infection in children: results of an 11-year randomized study. Clin Infect Dis 2007; 45: 715-722.

40 Tortajada C, Martinez-Lacasa J, Sanchez F, et al. Is the combination of pyrazinamide plus rifampicin safe for treating latent tuberculosis infection in persons not infected by the human immunodeficiency virus? Int J Tuberc Lung Dis 2005; 9: 276-281.

41 Sterling TR, Villarino ME, Borisov AS, et al. Three months of rifapentine and isoniazid for latent tuberculosis infection. N Engl J Med 2011; 365: 2155-2166.

42 Batki SL, Gruber VA, Bradley JM, et al. A controlled trial of methadone treatment combined with directly observed isoniazid for tuberculosis prevention in injection drug users. Drug Alcohol Depend 2002; 66: 283-293.

43 Malotte CK, Hollingshead JR, Larro M. Incentives vs outreach workers for latent tuberculosis treatment in drug users. Am J Prev Med 2001; 20: 103-107.

44 Tulsky J, Hahn J, Long H, et al. Can the poor adhere? Incentives for adherence to TB prevention in homeless adults. Int J Tuberc Lung Dis 2004; 8: 83-91.

45 White MC, Tulsky JP, Goldenson J, et al. Randomized controlled trial of interventions to improve follow-up for latent tuberculosis infection after release from jail. Arch Intern Med 2002; 162: 1044-1050.

46 Hirsch-Moverman Y, Colson P, Bethel J, et al. Can a peer-based intervention impact adherence to the treatment of latent tuberculous infection? Int J Tuberc Lung Dis 2013; 17: 1178-1185.

47 Hovell MF, Sipan CL, Blumberg EJ, et al. Increasing Latino adolescents' adherence to treatment for latent tuberculosis infection: a controlled trial. Am J Public Health 2003; 93: 1871-1877.

48 Kominski GF, Varon SF, Morisky DE, et al. Costs and cost-effectiveness of adolescent compliance with treatment for latent tuberculosis infection: results from a randomized trial. J Adolesc Health 2007; 40: 61-68.

49 Nyamathi A, Christiani A, Nahid P, et al. A randomized controlled trial of two treatment programs for homeless adults with latent tuberculosis infection. Int J Tuberc Lung Dis 2006; 10: 775-782.

50 Goldberg S, Wallace J, Jackson J, et al. Cultural case management of latent tuberculosis infection. Int J Tuberc Lung Dis 2004; 8: 76-82.

51 Sawert H, Girardi E, Antonucci G, et al. Preventive therapy for tuberculosis in HIV-infected persons: analysis of policy options based on tuberculin status and CD4+ cell count. Gruppo Italiano di Studio Tubercolosi e AIDS (GISTA). Arch Intern Med 1998; 158: 2112-2121.

52 Pooran A, Booth H, Miller RF, et al. Different screening strategies (single or dual) for the diagnosis of suspected latent tuberculosis: a cost effectiveness analysis. BMC Pulm Med 2010; 10: 7.

53 Burgos JL, Kahn JG, Strathdee SA, et al. Targeted screening and treatment for latent tuberculosis infection using QuantiFERON-TB Gold is cost-effective in Mexico. Int J Tuberc Lung Dis 2009; 13: 962-968.

54 Pareek M, Watson JP, Ormerod LP, et al. Screening of immigrants in the UK for imported latent tuberculosis: a multicentre cohort study and cost-effectiveness analysis. Lancet Infect Dis 2011; 11: 435-444.

55 Foster S, Godfrey-Faussett P, Porter J. Modelling the economic benefits of tuberculosis preventive therapy for people with HIV: the example of Zambia. AIDS 1997; 11: 919-925.

56 Schechter $\mathrm{CB}$, Rose DN, Fahs MC, et al. Tuberculin screening: cost-effectiveness analysis of various testing schedules. Am J Prev Med 1990; 6: 167-175.

57 Brassard P, Steensma C, Cadieux L, et al. Evaluation of a school-based tuberculosis-screening program and associate investigation targeting recently immigrated children in a low-burden country. Pediatrics 2006; 117: e148-e156.

58 Chang S, Wheeler LS, Farrell KP. Public health impact of targeted tuberculosis screening in public schools. Am J Public Health 2002; 92: 1942-1945.

59 Khan K, Muennig P, Behta M, et al. Global drug-resistance patterns and the management of latent tuberculosis infection in immigrants to the United States. N Engl J Med 2002; 347: 1850-1859. 
Linas BP, Wong AY, Freedberg KA, et al. Priorities for screening and treatment of latent tuberculosis infection in the United States. Am J Respir Crit Care Med 2011; 184: 590-601.

61 Oxlade O, Schwartzman K, Menzies D. Interferon-gamma release assays and TB screening in high-income countries: a cost-effectiveness analysis. Int J Tuberc Lung Dis 2007; 11: 16-26.

62 Currie CS, Floyd K, Williams BG, et al. Cost, affordability and cost-effectiveness of strategies to control tuberculosis in countries with high HIV prevalence. BMC Public Health 2005; 5: 130.

63 Linas BP, Wong AY, Freedberg KA, et al. Priorities for screening and treatment of latent tuberculosis infection in the United States. Am J Respir Crit Care Med 2011; 184: 590-601.

64 Dasgupta K, Schwartzman K, Marchand R, et al. Comparison of cost-effectiveness of tuberculosis screening of close contacts and foreign-born populations. Am J Respir Crit Care Med 2000; 162: 2079-2086.

65 Deuffic-Burban S, Atsou K, Viget N, et al. Cost-effectiveness of QuantiFERON-TB test vs. tuberculin skin test in the diagnosis of latent tuberculosis infection. Int J Tuberc Lung Dis 2010; 14: 471-481.

66 Tan M, Menzies D, Schwartzman K. Tuberculosis screening of travelers to higher-incidence countries: a cost-effectiveness analysis. BMC Public Health 2008; 8: 201.

67 Suthar AB, Lawn SD, del Amo J, et al. Antiretroviral therapy for prevention of tuberculosis in adults with HIV: a systematic review and meta-analysis. PLoS Med 2012; 9: e1001270.

68 World Health Organization. Guidance on Ethics of Tuberculosis Prevention, Care and Control. Geneva, World Health Organization, 2010.

69 Denholm JT, Matteelli A, Reis A. Latent tuberculous infection: ethical considerations in formulating public health policy. Int J Tuberc Lung Dis 2015; 19: 137-140.

70 Kass NE. An ethics framework for public health. Am J Public Health 2001; 91: 1776-1782.

71 World Health Organization. Systematic screening for active tuberculosis: principles and recommendations. Geneva, World Health Organization, 2013. 